\title{
Decision Fusion for Structural Damage Detection: Numerical and Experimental Studies
}

\author{
Yong Chen, ${ }^{1}$ Senyuan Tian, ${ }^{1}$ and Bingnan Sun ${ }^{1,2}$ \\ ${ }^{1}$ Department of Civil Engineering, Zhejiang University, Hangzhou, Zhejiang 310058, China \\ ${ }^{2}$ Ningbo Institute of Technology, Zhejiang University, Ningbo, Zhejiang 315100, China
}

Correspondence should be addressed to Yong Chen, cecheny@zju.edu.cn

Received 31 August 2009; Revised 28 December 2009; Accepted 23 March 2010

Academic Editor: Yi Qing Ni

Copyright () 2010 Yong Chen et al. This is an open access article distributed under the Creative Commons Attribution License, which permits unrestricted use, distribution, and reproduction in any medium, provided the original work is properly cited.

\begin{abstract}
This paper describes a decision fusion strategy that can integrate multiple individual damage detection measures to form a new measure, and the new measure has higher probability of correct detection than any individual measure. The method to compute the probability of correct selection is presented to measure the system performance of the fusion system that includes the presented fusion strategy. And parametric sensitive studies on system performance are also conducted. The superiority of the fusion strategy herein is that it can be extended to deal with the multiresolution subdecision or blind adaptive detection, and corresponding methodologies are also provided. Finally, an experimental setup was fabricated, whereby the vibration properties of damaged and undamaged structures were measured. The experimental results with the undamaged structural model provide information for producing an improved theoretical and numerical model via model updating techniques. Three existing vibration-based damage detection methods with varied resolutions were utilized to identify the damage that occurred in the structure, based on the experimental results. Then the decision fusion strategy was implemented to join the subdecisions from these three methods. The fused results are shown to be superior to those from single method.
\end{abstract}

\section{Introduction}

In the structural health monitoring (SHM) for civil engineering, the monitoring system that consists of numerous sensors is usually employed to achieve more accurate damage detection results, and a number of detection methods have been developed to identify the damages in accordance with structural vibration information (see, e.g., [1-5]). However, there is no perfect damage detection method that is capable of dealing with all kinds of structures, sensors or damages. Focusing on achieving a perfect single damage detection method to solve all damage detection problems does not sound feasible especially for civil engineering structures. Consequently, combining many detection methods together and fusing the sub-results to obtain more accurate detection results would be reasonable. Damage detection-oriented decision fusion that has this ability attracted increasing attentions of researchers studying SHM-based damage detection.

Actually, the initial data fusion researches were predominantly in the defense systems (see, e.g., [6]). It can be traced back to Tenney and Sandell's achievements [7], and it has been developed quickly in recent years. In early research, Chair and Varshney concentrated on binary decision fusion [8]. Demirbas provided a decision rule, based on maximizing the posteriori probability using binary detection results [9]. The work of Baek and Bommareddy brought the decision fusion research into a new field in which multiple hypotheses are for decision making [10]. Subsequently, blind adaptive decision fusion was developed to deal with the unknown system (see, e.g., [11-13]). Sinha et al. classified the modern decision fusion methods into five categories including linear opinion pool, log linear opinion pool, voting/ranking method, classifier fusion, and artificial neural net (ANN) fusion (see [6] for more details).

Similar as in fault diagnosis, image analysis, safety assessment, and others (see, e.g., [14-16]), many beneficial and novel studies on applying decision fusion method were conducted in structural damage detection. It is found that decision fusion can significantly improve the reliability and robustness of monitoring system through fusing multiple 
damage detection results. Dempster-Shafer (D-S) evidential theory has been proved to be a powerful information fusion strategy, many studies on its application to structural damage detection have been conducted. Guo and Zhang applied a weighted balance evidence theory to identifying the damages of a two dimensional truss structure [17]. Zhou et al. utilized D-S evidential theory to merge the multiresolution decision results [18]. Li et al. combined the ANN detection methods and Dempster-Shafer evidential theory together to identify the damages in civil structures, and ANN was used to obtain sub-decision results [19]. In respect of statistical inference, the effort is also seen. Tshöpe and wolff developed a self-learning method which applies classifier in order to identify objects or assess their states, and this method was experimentally verified in damage identification of an aluminum plate and a carbon fiber reinforced plastic plate [20]. Based on Bayesian probability principles, Tian et al. provided a statistical decision fusion methodology, defined as maximum joint probability (MJP) decision rule for damage detection [21]. With regard to comparison of decision fusion strategies, Wang et al. applied four decision fusion strategies, including voting scheme, Bayesian inference, D-S evidential theory, and fuzzy inference, to a damage detection case of a plate structure, and the corresponding experimental studies were also conducted [22].

In this paper, we introduced extended utilizations of MJP decision rule to deal with the multiresolution decision fusion and adaptive decision fusion. With this method, a fusion scheme was proposed for experimental damage detection. Firstly, a cantilever beam was experimentally set up in laboratory and numerically modeled by finite element method (FEM), and the FEM model was updated by the use of experimentally obtained vibration information of undamaged beam. By the use of updated numerical model, damages were simulated numerically, and corresponding natural frequencies were calculated. Noises were added to the natural frequencies for simulating the experimental error. Then, using these numerical results, the probabilities of correct selection of three damage detection methods were obtained. Subsequently, the experimentally acquired vibration information of the damaged physical beam was treated as input of three damage detection methods. Thus, the detection results and priori probabilities obtained in advance were integrated by decision fusion rules to attain a final detection results. The probabilities of correct selection of this fusion system were also obtained, and superiority was found while comparing with that of single damage detection system.

\section{Damage Detection Oriented Decision Fusion}

Multiple damage cases and multiresolution damage detection requirements are the predominant factors in applying the decision fusion rules to damage detection. This section provides extended forms of MJP decision fusion strategy to deal with those difficulties. For convenience of theoretical expression, it is noted that the concepts of hypothesis, sub-decision system, and decisions those expressions in statistical theory have the same meaning as damage case, damage detection method (detector or damage detector), and damage detection results, respectively.

2.1. MJP Fusion Rule. MJP fusion rule (see [21] for more details) is trying to make a decision that is capable of minimizing the Bayesian risk function based on Bayesian probability theory. It is assumed that there exist $M+1$ hypotheses, which can be represented by variable $\mathbf{H}=$ $\left\{H_{0}, H_{1}, H_{2}, \ldots, H_{M}\right\}$, and $P\left(H_{j}\right)$ refers to the priori probability of the $j$ th hypothesis. It is also assumed that there exist $N$ damage detectors. The sub-decision made by each detector can be designated as $u_{i}(i=1, \ldots, N)$, and all of sub-decisions would form a decision state vector $\mathbf{u}=\left\{u_{1}, u_{2}, \ldots, u_{N}\right\}$. For the $i$ th detector, the description of its performance can be represented by a conditional probability matrix $\mathbf{h}^{i}$, in which each element is actually a conditional probability and has a form of

$$
\begin{array}{r}
h_{j k}^{i}=P\left(u_{i}=h_{j} \mid H_{k}\right), \\
i=1, \ldots, N, \quad j=0,1, \ldots, M, \quad k=0,1, \ldots, M,
\end{array}
$$

where $h_{j k}^{i}$ is the probability of the case that the $j$ th hypothesis is selected by the $i$ th detector while the $k$ th hypothesis occurs, and $h_{j}$ is the $j$ th hypothesis selected by detector.

By the use of the Bayesian risk function (see [23] for more details) and the definition of the final decision $f(\mathbf{u})=h_{B}$ in which $B \in\{0,1, \ldots, M\}$, we have Bayesian risk as

$$
R_{P}(f \mid \mathbf{u})=\mathrm{E}_{H \mid \mathbf{u}} L(H, f)=\sum_{k=0}^{M} L\left(H_{k}, f\right) P\left(H_{k} \mid \mathbf{u}\right),
$$

in which the decision rule $f(\mathbf{u})$ yields a final decision $f(\mathbf{u})=$ $h_{B}$ that leads to a minimum Bayesian risk. $L\left(H_{k}, f(\mathbf{u})\right)$ is the Loss Function defined as

$$
L\left(H_{k}, f(\mathbf{u})=h_{j}\right)= \begin{cases}0, & \text { if } k=j \\ 1, & \text { otherwise }\end{cases}
$$

Substituting (3) into (2) yields

$$
\begin{aligned}
R_{P}\left(f=h_{B} \mid \mathbf{u}\right) & =\min R_{P}(f \mid \mathbf{u}) \\
& =\min _{j \in\{0,1, \ldots, M\}}\left[\sum_{k=0}^{M} L\left(H_{k}, u=h_{j}\right) P\left(H_{k} \mid \mathbf{u}\right)\right] \\
& =\sum_{k=0}^{M} P\left(H_{k} \mid \mathbf{u}\right)-\max _{k \in\{0,1, \ldots, M\}}\left[P\left(H_{k} \mid \mathbf{u}\right)\right] \\
& =1-\max _{k \in\{0,1, \ldots, M\}}\left[P\left(H_{k} \mid \mathbf{u}\right)\right] .
\end{aligned}
$$

Accordingly, if a $P\left(H_{B} \mid \mathbf{u}\right)$ is the largest value among all $P\left(H_{k} \mid \mathbf{u}\right)$, thus the final decision $f(\mathbf{u})=h_{B}$ is the selection that minimizes the Bayesian cost function. This decision process could be denoted as

$$
f(\mathbf{u}) \triangleq \max _{k \in\{0,1, \ldots, M\}}^{-1}\left[P\left(H_{k} \mid \mathbf{u}\right)\right] .
$$


It is also assumed that the detectors work independently. Thus, the joint probability of combined decision state can be obtained by

$$
P\left(\mathbf{u} \mid H_{k}\right)=\prod_{i=1}^{N} P\left(u_{i} \mid H_{k}\right) .
$$

With consideration that $P(\mathbf{u})$ is a constant, using Bayesian rule, (6) leads to a new form of the decision rule, that is,

$$
\begin{aligned}
f(\mathbf{u}) & \triangleq \max _{k \in\{0,1, \ldots, M\}}\left[P\left(H_{k}\right) \prod_{i=1}^{N} P\left(u_{i} \mid H_{k}\right)\right] \\
& =\max _{k \in\{0,1, \ldots, M\}} I_{k},
\end{aligned}
$$

in which $I_{k}=P\left(H_{k}\right) \prod_{i=1}^{N} P\left(u_{i} \mid H_{k}\right)$ is defined as the picking index of hypothesis $H_{k}$ under determined u. Equation (7) also means that the decision rule derived herein is to select a hypothesis that maximizes the joint probability of all detectors' decisions,

According to (7), the final decision $f(\mathbf{u})=h_{B}$ satisfies

$$
\frac{P\left(H_{B}\right) \prod_{i=1}^{N} P\left(u_{i} \mid H_{B}\right)}{P\left(H_{l}\right) \prod_{i=1}^{N} P\left(u_{i} \mid H_{l}\right)} \geq 1, \quad l \in\{0,1, \ldots, M\}, l \neq B,
$$

that is,

$\ln \frac{P\left(H_{B}\right)}{P\left(H_{l}\right)}+\sum_{i=1}^{N} \ln \frac{P\left(u_{i} \mid H_{B}\right)}{P\left(u_{i} \mid H_{l}\right)} \geq 0, \quad l \in\{0,1, \ldots, M\}, l \neq B$.

The picking coefficient $v_{j}^{i}$ for $i$ th detector whose decision is $h_{g}$ can be represented as

$$
v_{j}^{i}=\left\{\begin{array}{ll}
1 & \text { if } j=g, \\
0 & \text { otherwise. }
\end{array} \quad j=0,1, \ldots, M .\right.
$$

Using the weight factors denoted as

$$
\begin{gathered}
w_{k, l}=\ln \frac{P\left(H_{k}\right)}{P\left(H_{l}\right)}, \\
w_{i, j, k, l}=\ln \frac{P\left(u_{i}=h_{j} \mid H_{k}\right)}{P\left(u_{i}=h_{j} \mid H_{l}\right)}
\end{gathered}
$$

and the picking coefficients also leads to a new form of the decision rule, that is, the final decision $f(\mathbf{u})=h_{B}$ is the hypothesis that satisfies

$$
\sum_{\substack{l=1 \\ l \neq B}}^{M} \operatorname{sign}\left(y_{B, l}\right)=M
$$

where

$$
y_{k, l}=w_{k, l}+\sum_{i=1}^{N} \sum_{j=0}^{M} v_{j}^{i} w_{i, j, k, l}
$$

and the definition of $\operatorname{sign}(\cdot)$ is

$$
\operatorname{sign}(x)= \begin{cases}1 & \text { if } x \geq 0 \\ 0 & \text { otherwise }\end{cases}
$$

As well as (7), (12) is also another form of decision rule, and it is easy to be utilized while considering an adaptive fusion problem. Summarily, the decision rule derived herein is to achieve the maximum joint probability of the subdecisions state $\left\{u_{1}, u_{2}, \ldots, u_{n}\right\}$ while an actual unknown damage case occurs. Accordingly, it was defined as maximum joint probability (MJP) decision rule (see [21]).

2.2. Probability of Correct Selection. It is necessary to be aware of the probability of correct selection (PCS) of the whole fusion system. According to the definition of the Loss Function in (4), the Bayesian risk function presented by (3) is actually the probability of system making a wrong decision. Therefore, the probability of failure (PF) for a decision made according to MJP decision rule (see [21]) is

$$
W\left(f(\mathbf{u})=u_{B} \mid \mathbf{u}\right)=1-P\left(H_{B}\right) \frac{\prod_{i=1}^{N} P\left(u_{i} \mid H_{B}\right)}{P(\mathbf{u})},
$$

where

$$
P(\mathbf{u})=\sum_{k=1}^{M} P\left(\mathbf{u} H_{k}\right) P\left(H_{k}\right)=\sum_{k=1}^{M} P\left(H_{k}\right) \prod_{i=1}^{N} P\left(u_{i} \mid H_{k}\right)
$$

and the PCS is

$$
Q\left(f(\mathbf{u})=u_{B} \mid \mathbf{u}\right)=1-W\left(f(\mathbf{u})=u_{B} \mid \mathbf{u}\right) .
$$

For the entire decision system in association with decision rule $f$, the PF and PCS of the whole system, denoted as $W(f)$ and $Q(f)$, respectively, are formed (see [21]) as

$$
\begin{gathered}
W(f)=\sum_{\mathbf{u}} W\left(f(\mathbf{u})=u_{B} \mid \mathbf{u}\right) P(\mathbf{u}), \\
Q(f)=1-W(f) .
\end{gathered}
$$

2.3. Multiresolution. Damage detection results can be conventionally classified into three levels: damage acknowledgement, damage localization and damage quantification (see [5]). Variable $l$ is used to represent these three levels of resolution. The damage detection method on the first level $(l=1)$ is able to determine whether the damage occurred or not. If a damage detection method is on the second level $(l=2)$, it means that this method can alarm not only the occurrence of damage but also the location of damage. The third level $(l=3)$ is the highest level, and on this level the damage detection method can provide both localization and quantification of a damage.

Figure 1 illustrates an application of the definition of resolution. The bridge in Figure 1 consists of three segments, and there are two grades of damage quantity to be identified. Thus, in case of the lowest level, the hypotheses for decision fusion are Damaged and No Damage. For the second level, the hypotheses for decision fusion would be No Damage and 


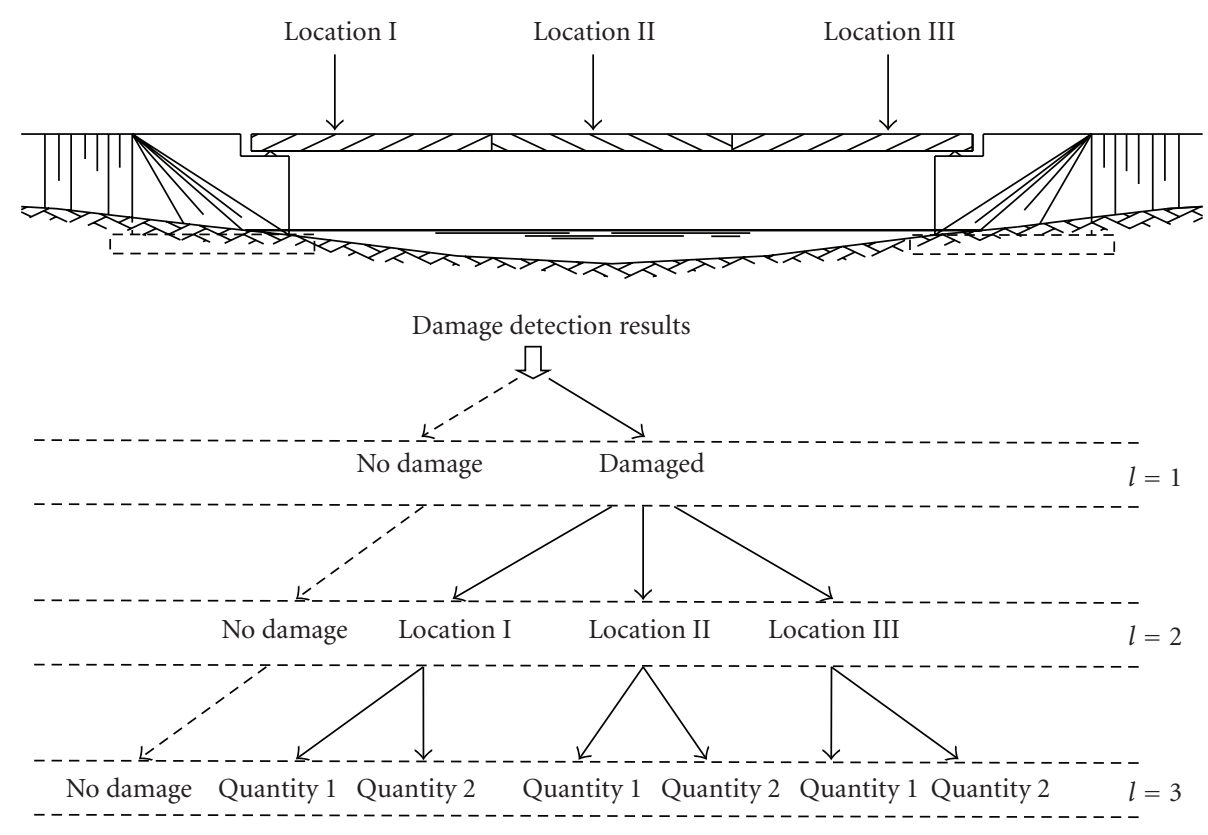

FIgURE 1: Three damage resolutions of damage detection methods.

Damaged-Location I, Damaged-Location II, and DamagedLocation III. With respect to the highest level, a total of seven hypotheses would be No Damage, Damaged-Location I-Quantity 1, Damaged-Location I-Quantity 2, DamagedLocation II-Quantity 1, Damaged-Location II-Quantity 2, Damaged-Location III-Quantity 1, and Damaged-Location III-Quantity 2. For example, we assume that the damage occurred in the second segment with a damage degree that is Quantity 2. We also assume that three damage detection methods are on different resolution levels, and they are sorted by resolution from the lowest to highest. If the detectors detected the damage correctly, the results from these three damage detection methods would sequentially be Damaged, Damaged-Location II, and Damaged-Location IIQuantity 2. For convenience, the hypotheses are conventionally numbered sequentially from zero, and a correlation table is prepared to describe the relationship between the number and hypothesis. Thus, the detection results in this example should be 1,2 and 4 sequentially.

In terms of multiresolution, the hypotheses or damage cases are determined by the damage detection method that is on the highest resolution level among all detectors. With a consideration of a detector being on the highest level $(l=$ $3)$, the damage case vector $\left\{h_{0}^{l=3}, h_{1}^{l=3}, \ldots, h_{M}^{l=3}\right\}$ has a size of $M=s \times r$, in which $s$ and $r$ represent the number of damage locations and grades, respectively. For example, if considering the problem in Figure 1, then $s$ is 3 and $r$ is 2 . Hence, on the reduced level $(l=2)$ of resolution for the same detection method, the damage cases for identifying should be No Damage and the damage localizations, that is, $\left\{h_{0}^{l=2}, h_{1}^{l=2}, \ldots, h_{s}^{l=2}\right\}$. Also, they would be No Damage and Damaged, that is, $\left\{h_{0}^{l=1}, h_{1}^{l=1}\right\}$, while the resolution is reduced again to the first level $(l=1)$. The resolution level, that is, $l=1,2$ or 3 , is marked as a superscript of the sub-decision, as well as the detectors and the hypotheses. For instance, $u_{2}^{l=2}$ means that the second detection method is on level 2.

A damage detection method with the highest level $(l=3)$ resolution can be reduced to a method on the level of $l=2$. The corresponding conditional probability $P\left(u_{i}^{l=2}=h_{q}^{l=2}\right.$ | $\left.H_{k}\right)$ of the latter can be computed through $P\left(u_{i}^{l=3}=h_{j}^{l=3}\right.$ | $H_{k}$ ) of the former, that is,

$$
\begin{aligned}
h_{q k}^{i}= & P\left(u_{i}^{l=2}=h_{q}^{l=2} \mid H_{k}\right) \\
& = \begin{cases}\sum_{j=(q-1) r+1}^{q r} P\left(u_{i}^{l=3}=h_{j}^{l=3} \mid H_{k}\right) & \text { if } q=1, \ldots, s, \\
P\left(u_{i}^{l=3}=h_{0}^{l=3} \mid H_{k}\right) & \text { if } q=0 .\end{cases}
\end{aligned}
$$

Similarly, if a method's resolution is reduced from level 2 to level 1, the new conditional probabilities $P\left(u_{i}^{l=1}=h_{0}^{l=1}\right.$ | $\left.H_{k}\right)$ and $P\left(u_{i}^{l=1}=h_{1}^{l=1} \mid H_{k}\right)$ for the lowest resolution level would be

$$
\begin{aligned}
& h_{1 k}^{i}=P\left(u_{i}^{l=1}=h_{1}^{l=1} \mid H_{k}\right)=\sum_{q=1}^{s} P\left(u_{i}^{l=2}=h_{q}^{l=2} h_{q} \mid H_{k}\right), \\
& h_{0 k}^{i}=P\left(u_{i}^{l=1}=h_{0}^{l=1} \mid H_{k}\right)=P\left(u_{i}^{l=2}=h_{0}^{l=2} \mid H_{k}\right) .
\end{aligned}
$$

According to the maximum joint probability decision rule, for a multiresolution case, optimal final decision is also the one that achieves a maximum joint probability of sub-decisions made by detection methods with different resolutions. For example, three sub-decisions are made but on different resolution levels. If the sub-decisions for $k$ th 
hypothesis are $u_{1}^{l=1}=h_{1}^{l=1}, u_{2}^{l=2}=h_{2}^{l=2}$, and $u_{3}^{l=3}=h_{2}^{l=3}$, thus the picking index in (7) is

$$
\begin{aligned}
I_{k}= & P\left(H_{k}\right) \prod_{i=1}^{N} P\left(u_{i} \mid H_{k}\right) \\
= & P\left(u_{1}^{l=1}=h_{1}^{l=1} \mid H_{k}\right) P\left(u_{2}^{l=2}=h_{2}^{l=2} \mid H_{k}\right) \\
& \times P\left(u_{3}^{l=3}=h_{2}^{l=3} \mid H_{k}\right) P\left(H_{k}\right) \\
= & h_{1 k}^{1} h_{2 k}^{2} h_{2 k}^{3} P\left(H_{k}\right)
\end{aligned}
$$

Then we can vary the hypothesis and make a comparison, and the hypothesis corresponding to the maximum one is the final decision.

Multiresolution decision fusion strategy can be carried out on the reduced level, by taking advantage of (20) and (21) and modifying the value range of $k$ in (7) by

$$
k \in \begin{cases}\{0,1, \ldots, M\} & \text { if } l=3, \\ \{0,1, \ldots, s\} & \text { if } l=2 \\ \{0,1\} & \text { if } l=1\end{cases}
$$

\section{Parametric Study in Same Resolution Case}

In the parametric study for system performance, it is assumed that all of the detectors have the same resolution. Also, the priori probability of each damage case is known as

$$
P\left(H_{k}\right)=\frac{1}{(M+1)} \quad k=0,1, \ldots, M,
$$

where $M$ represents number of damage cases. The probability of correct selection by damage detection method numbered $i$ is

$$
Q_{i}=\sum_{j=0}^{M} P\left(u_{i}=h_{j} \mid H_{j}\right) P\left(H_{j}\right)
$$

It is also assumed that each damage detection method has the same performance which means that they have the same PCS, that is, all $P\left(u_{i}=h_{j} \mid H_{j}\right)$ of the detectors are equal to $Q_{0}$. And all $P\left(u_{i}=h_{j} \mid H_{k}\right)$ in which $j \neq k$ are equal for each damage detection methods. Summarily, the conditional probability matrixes of the detectors are the same, and the elements in the matrixes are

$$
\begin{aligned}
& h_{j k}^{i}=P\left(u_{i}=h_{j} \mid H_{k}\right)= \begin{cases}Q_{0} & \text { if } j=k \\
\frac{\left(1-Q_{0}\right)}{M} & \text { otherwise }\end{cases} \\
& i=1, \ldots, N ; \quad j=0,1, \ldots, M ; \quad k=0,1, \ldots, M .
\end{aligned}
$$

Following Figures 2, 3, and 4 are based on these assumptions represented by (24) and (26), and the PCS of fusion system can be computed through (19).

Figure 2 illustrates the curves of the PCS of fusion system versus that of sub-decision system. In case of using five damage cases as shown in Figure 2(a), it is found that the
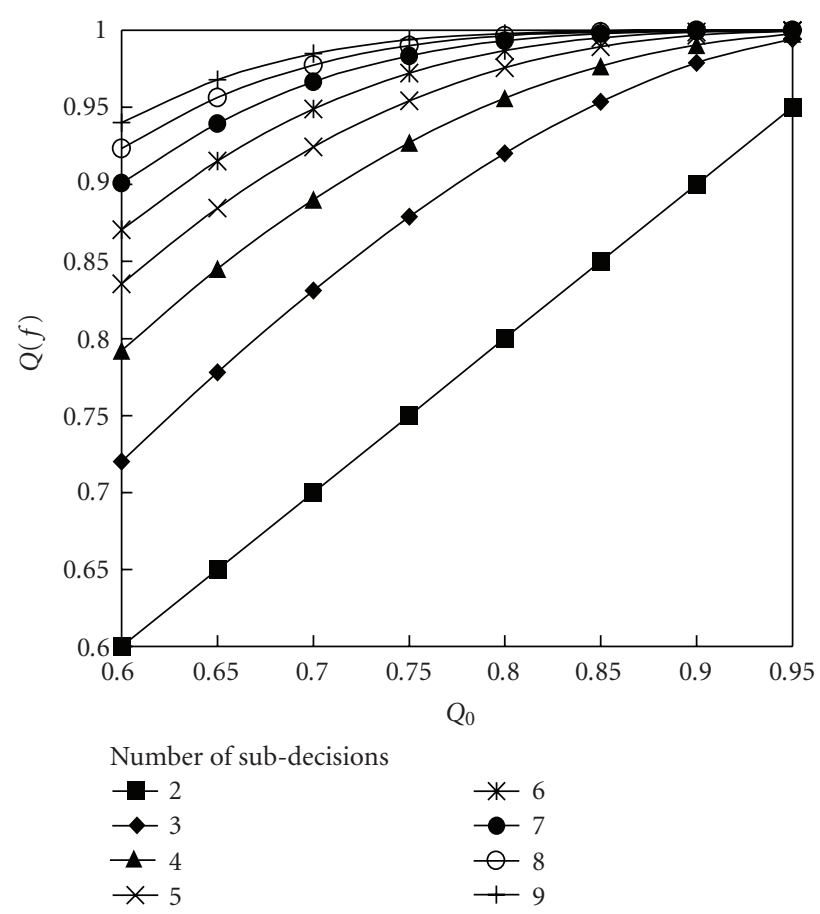

(a) Use five damage cases

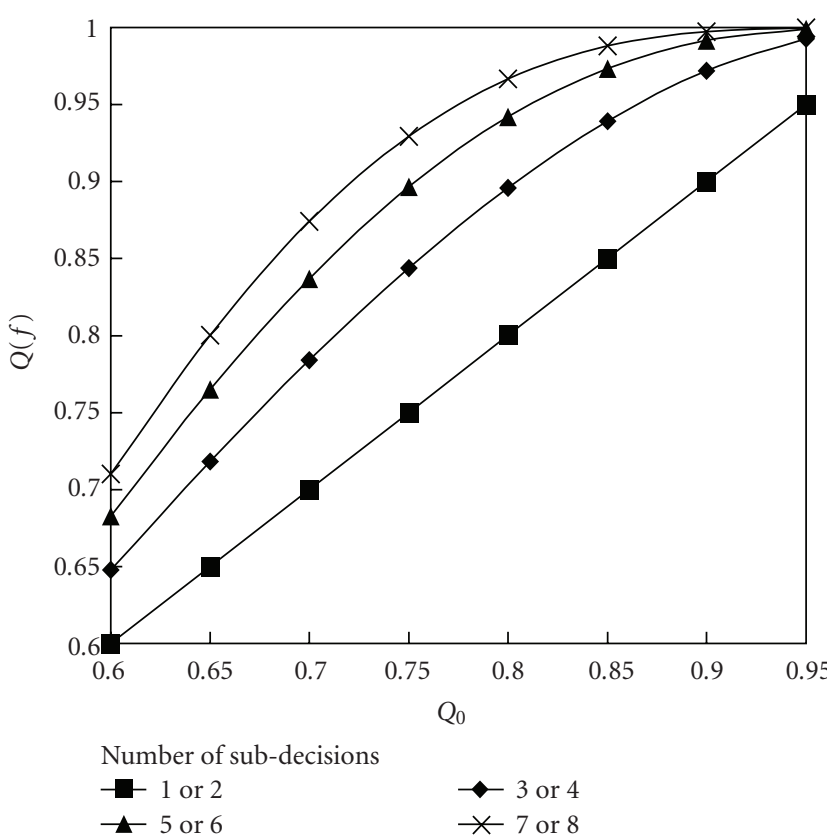

(b) Use two damage cases

Figure 2: PCS of fusion system versus that of sub-decision system.

PCS of the fusion system is improved while we increase the number of sub-decision methods and the PCS of the sub-decision systems. Also, the increment descends while adding supplemental sub-decision methods. In case of using two damage cases as shown in Figure 2(b), the similar phenomenon is observed. It is also found that using either $2 k-1$ or $2 k$ sub-decisions will achieve the same PCS of the fusion system, and $k=0,1, \ldots$. 
The PCS of the sub-decisions in Figure 3 is fixed at 0.8 . It is found that if the number of the sub-decision systems exceeds three, the PCS of the fusion system is significantly improved, and it increases with the increasing of the number of the damage cases. If we fix the number of sub-decisions at four, Figure 4 also indicates that increasing the number of the damage cases is beneficial to improve the PCS of the fusion system.

\section{Adaptive Decision Fusion}

4.1. Algorithm. For an unknown system, the knowledge of performance of the sub-decision systems, that is, conditional probabilities, is not available, as well as the priori probabilities of the hypotheses. That means the weight factors, which are necessary for the decision fusion rule formed as (7) or (13), are not available. Adaptive decision fusion strategy has capability of dealing with that. A simple counting rule-based (see, e.g., $[11,12]$ ) adaptive strategy for MJP are introduced herein to make a final decision by self learning and sensing the system continually.

In accordance with the simple counting rule, relative frequency (see, e.g., $[11,12]$ ), which is the ratio of the numbers of two events, is utilized to evaluate the weight factors. Since the true hypothesis is unknown for fusion system, we use a reliable hypothesis to replace the true hypothesis. This reliable hypothesis during the whole adaptive fusion procedure can be the final decision that is made by the decision fusion system, and we put a notation " $\wedge$ " above $H$ to distinguish the reliable hypothesis from the true hypothesis. Accordingly, notation " $\wedge$ " marked weight factors are used to indicate that they are based on the reliable hypothesis. Let $a_{k}$ be the number of $\hat{H}_{k}, a_{l}$ be the number of $\hat{H}_{l}, a_{i, j, k}$ be the number of decisions that is the $j$ th hypothesis which is made by the $i$ th detector while $\hat{H}_{k}$ occurs, and $a_{i, j, l}$ be the number of decisions that is the $j$ th hypothesis which is made by the $i$ th detector while $\hat{H}_{l}$ occurs, thus the weight factors in (13) can be approximately estimated by

$$
\begin{gathered}
\hat{w}_{k, l}=\ln \frac{P\left(\hat{H}_{k}\right)}{P\left(\hat{H}_{l}\right)} \approx \ln \frac{a_{k}}{a_{l}}, \\
\hat{w}_{i, j, k, l}=\ln \frac{P\left(u_{i}=h_{j}, \hat{H}_{k}\right)}{P\left(u_{i}=h_{j}, \hat{H}_{l}\right)}-\ln \frac{P\left(\hat{H}_{k}\right)}{P\left(\hat{H}_{l}\right)} \approx \ln \frac{a_{i, j, k}}{a_{i, j, l}}-\widehat{w}_{k, l},
\end{gathered}
$$

whereby, we have

$$
\begin{gathered}
a_{l} \approx \frac{a_{k}}{\exp \left(\widehat{w}_{k, l}\right)}, \\
a_{i, j, l} \approx \frac{a_{i, j, k}}{\exp \left(\hat{w}_{i, j, k, l}+\hat{w}_{k, l}\right)} .
\end{gathered}
$$

Then, the partial derivatives of the weight factors are

$$
\begin{gathered}
\frac{\partial \hat{w}_{k, l}}{\partial a_{k}} \approx \frac{1}{a_{k}}, \\
\frac{\partial \hat{w}_{k, l}}{\partial a_{l}} \approx-\frac{1}{a_{l}} \approx-\frac{1}{a_{k}} \exp \left(\widehat{w}_{k, l}\right), \\
\frac{\partial \hat{w}_{i, j, k, l}}{\partial a_{k}} \approx-\frac{1}{a_{k}}, \\
\frac{\partial \hat{w}_{i, j, k, l}}{\partial a_{l}} \approx \frac{1}{a_{l}} \approx \frac{1}{a_{k}} \exp \left(\widehat{w}_{k, l}\right), \\
\frac{\partial \hat{w}_{i, j, k, l}}{\partial a_{i, j, k}} \approx \frac{1}{a_{i, j, k}}, \\
\frac{\partial \hat{w}_{i, j, k, l}}{\partial a_{i, j, l}} \approx-\frac{1}{a_{i, j, l}} \approx-\frac{1}{a_{i, j, k}} \exp \left(\widehat{w}_{i, j, k, l}+\widehat{w}_{k, l}\right) .
\end{gathered}
$$

By substitution of (29) into the increment form of (27), the updating rule for weight factors can be

$$
\begin{gathered}
\hat{w}_{k, l}^{+}=\widehat{w}_{k, l}^{-}+\Delta \widehat{w}_{k, l} \\
\widehat{w}_{i, j, k, l}^{+}=\widehat{w}_{i, j, k, l}^{-}+\Delta \widehat{w}_{i, j, k, l},
\end{gathered}
$$

where

$$
\begin{aligned}
\Delta \hat{w}_{k, l} & = \begin{cases}\frac{1}{a_{k}} & \hat{H}=\hat{H}_{k}, \\
-\frac{1}{a_{k}} \exp \left(\hat{w}_{k, l}^{-}\right) & \hat{H}=\hat{H}_{l}, \\
0 & \hat{H} \neq \hat{H}_{k}, \hat{H} \neq \hat{H}_{l},\end{cases} \\
\Delta \hat{w}_{i, j, k, l} & = \begin{cases}-\frac{1}{a_{k}}+\frac{1}{a_{i, j, k}} & \hat{H}=\hat{H}_{k}, u_{i}=h_{j}, \\
-\frac{1}{a_{k}} & \hat{H}=\hat{H}_{k}, u_{i} \neq h_{j}, \\
\frac{1}{a_{k}} \exp \left(\hat{w}_{k, l}^{-}\right) & \hat{H}=\hat{H}_{l}, u_{i}=h_{j}, \\
-\frac{1}{a_{i, j, k}} \exp \left(\hat{w}_{i, j, k, l}^{-}+\hat{w}_{k, l}^{-}\right) & \hat{H}=\hat{H}_{l}, u_{i} \neq h_{j}, \\
\frac{1}{a_{k}} \exp \left(\hat{w}_{k, l}^{-}\right) & \hat{H} \neq \hat{H}_{k}, \hat{H} \neq \hat{H}_{l}, \\
0 & \end{cases}
\end{aligned}
$$

in which the superscript "+" means the current step, and “-” the previous step.

4.2. Numerical Simulation Study. To justify the efficiency of the proposed adaptive fusion methodology, a three hypothesis problem was numerically studied. It is assumed that the true priori probabilities of hypotheses are listed as

$$
P\left(H_{0}\right)=0.4, \quad P\left(H_{1}\right)=0.2, \quad P\left(H_{2}\right)=0.4 .
$$


It is also assumed that there are four independently working detectors. The true conditional probability matrices of these four sub-decision systems are assumed to be

$$
\begin{array}{rlrl}
\mathbf{h}^{1} & =\left[\begin{array}{lll}
0.90 & 0.04 & 0.06 \\
0.05 & 0.90 & 0.04 \\
0.05 & 0.06 & 0.90
\end{array}\right], & \mathbf{h}^{2}=\left[\begin{array}{lll}
0.82 & 0.07 & 0.08 \\
0.09 & 0.85 & 0.09 \\
0.09 & 0.08 & 0.83
\end{array}\right], \\
\mathbf{h}^{3}=\left[\begin{array}{lll}
0.91 & 0.04 & 0.05 \\
0.05 & 0.91 & 0.05 \\
0.04 & 0.05 & 0.90
\end{array}\right], & \mathbf{h}^{4}=\left[\begin{array}{lll}
0.85 & 0.07 & 0.08 \\
0.08 & 0.86 & 0.08 \\
0.07 & 0.07 & 0.84
\end{array}\right] .
\end{array}
$$

For adaptive fusion system, the true conditional probability matrices and priori probabilities of hypotheses are unknown. Equations (32), (33) are merely utilized to generate the events and corresponding sub-decisions, as input data of adaptive fusion system. A total of 10000 random events were generated according to (32). Based on these events, a total of 10000 sets of sub-decisions are also individually simulated according to (33). Each set of them consists of four pseudo sub-decisions on the corresponding event. Thus, by using the fusion strategy and (30), the adaptation of the weight factors can be figured out.

Figures 5 and 6 illustrate the adaptations of selected two weight factors, and compare them with true values. In the figures, the true values computed by (27) are horizontal straight lines and the true values are pointed out by variables without " $\wedge$ ". As shown in Figures 5 and 6 , the convergence of these curves justifies the effectiveness of the adaptive strategy we proposed. However, a very slow speed of convergence is observed, which is not suitable for civil structural damage detection. It is necessary to improve the speed of convergence in the future study.

\section{Experimental Study}

In this section, the framework of using decision fusion is introduced. We used two models for the same test structure, one is physical model for test and the other one is numerical model for performance study of detectors. The fusion scheme can be described as follows.

Step 1. Obtain the priori probabilities of hypotheses and conditional probability matrices of detectors;

Step 2. Detectors make sub-decisions based on the test results of actual structure.

Step 3. By the use of decision fusion, provide a final decision to determine the damage which occurred in actual structure.

The work in Step 1 should be completed in advance before starting damage detection, and it requires a lot of computational time and data preparation for civil engineering structures. The priori probabilities of hypotheses can be approximately obtained by taking advantage of expert experience or lots of statistical inspections of the existing

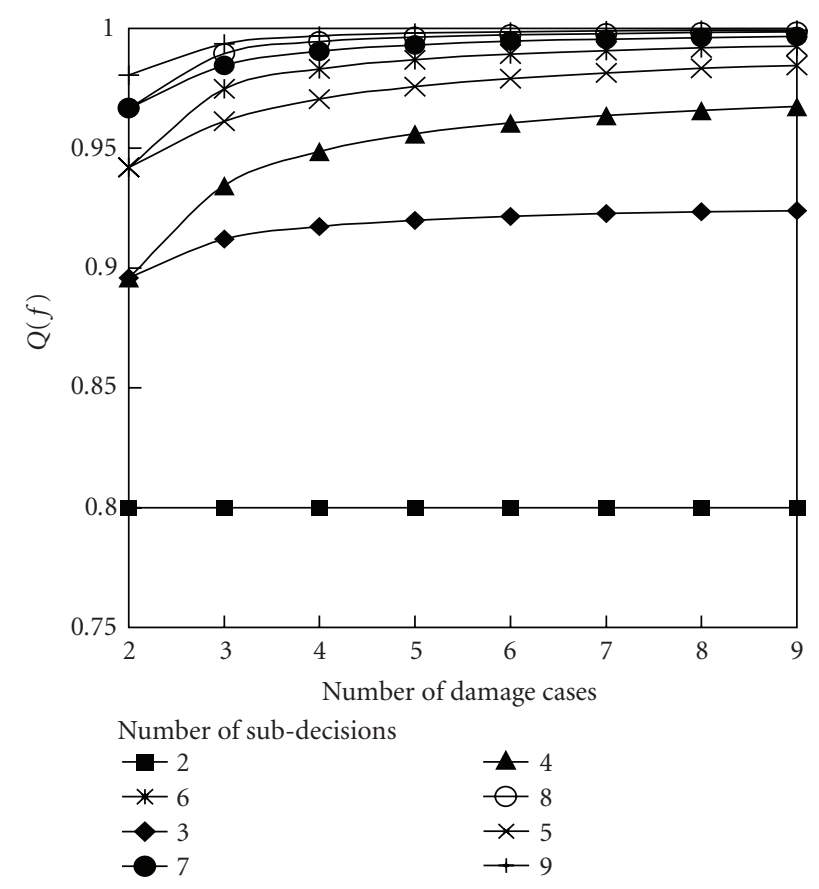

Figure 3: PCS of system versus number of damage cases in case of $Q_{0}=0.8$.

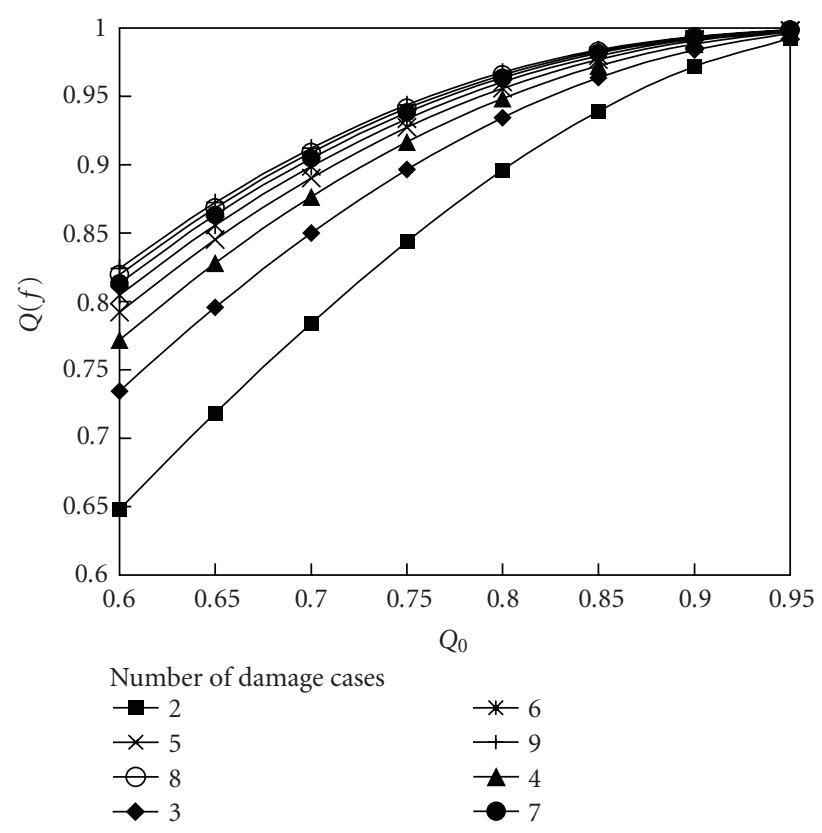

FIGURE 4: PCS of system versus that of sub-decision system, number of sub-decisions is four.

structures that considered congeneric with the test structure. The conditional probability matrixes of the detectors can be acquired according to the experience from applications, or by supplier.

We also introduce a countermeasure to obtain the conditional probability matrixes based on numerical damages that are simulated by using finite element analysis (FEA) method. 


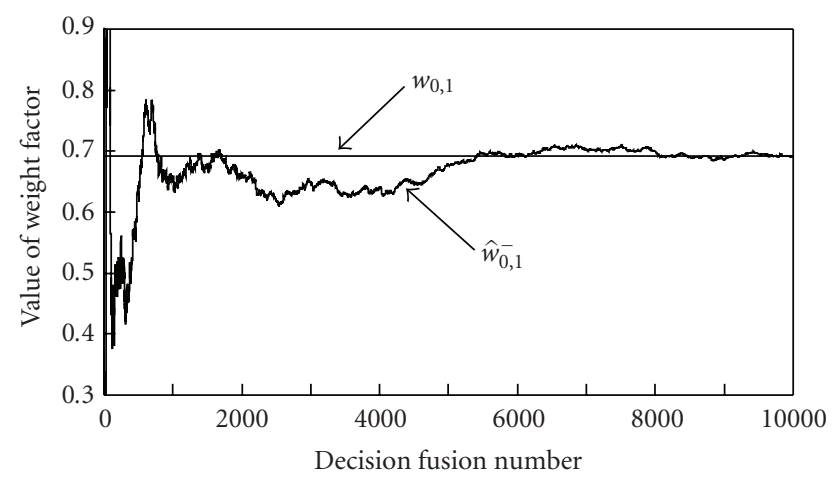

FIGURE 5: Adaptation of $\widehat{w}_{0,1}^{-}$.

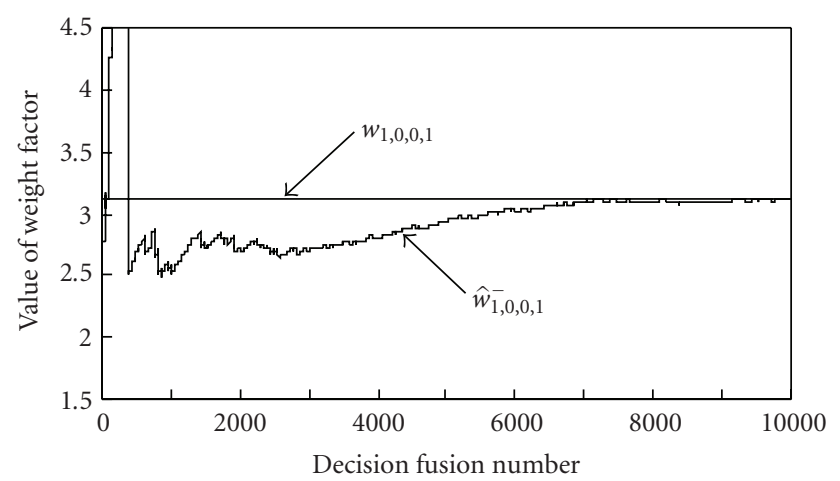

Figure 6: Adaptation of $\widehat{w}_{1,0,0,1}^{-}$.

At first, numerical damage cases were simulated by the use of FEA method, and corresponding natural frequencies of undamaged and damaged structures were calculated. It is well known that the failure in detecting is due to the test noise. Therefore, we added a numerical white noise on the natural frequencies that are calculated by FEA. The noise level is the same as that of the actual measuring system, and the actual noise can be obtained by repeated tests on the actual structure. Thus, numerous noised samples were obtained. Whereby, all of the detectors are examined to detect the numerical simulated damage. For each detector, by taking statistical analysis on the detection results on all events, it is easy to approximately achieve conditional probability matrixes.

Damage detections in Step 2 are a kind of blind damage detection, since the structural damage is unknown. The detection results made by the detectors may agree or disagree with each other, and each detection result is provided with a probability of correct selection. If we stop at this step, the only reasonable selection is the one provided by the detector that has the highest PCS. If we go on, and fuse these detection results by decision fusion rule with the priori probabilities and the conditional probability matrixes in Step 1, then a final decision that has a higher PCS will be obtained.

Regarding the experimental application presented in this section, Figure 7 illustrates the corresponding strategy for damage detection, which is based on decision fusion. The strategy utilized three vibration-based (actually natural
TABLE 1: Natural frequencies of cantilever beam $(\mathrm{Hz})$.

\begin{tabular}{lccccc}
\hline Order & 1 & 2 & 3 & 4 & 5 \\
\hline Experimental results & 7.39 & 45.46 & 128.6 & 256.5 & 415.8 \\
Numerical results & 7.66 & 47.44 & 134.0 & 263.5 & 433.9 \\
After model updating & 7.39 & 45.46 & 128.5 & 253.0 & 414.6 \\
\hline
\end{tabular}

frequency-based) damage detection methods as sub-decision systems. Since the detection methods employed herein are different in resolution, we use a multiresolution fusion rule to carry out the decision fusion.

5.1. Test Structure. The specimen for the experimental study is a steel cantilever beam with $1 \mathrm{~m}$ in length as shown in Figure 8. Three accelerometers SA1, SA2 and SA3, were installed at different positions of the structure to measure the accelerations of the beam. The location of excitation is EP in Figure 8, where force hammer was used to give an impulse excitation.

The vibrations under impulse excitation were measured to identify the natural frequencies of undamaged structure. The identified natural frequencies are also compared with FEA results, as shown in Table 1. Though a small error between numerical and experimental results is found, it would not be good enough for damage detection. This is because the damage detection methods used herein merely rely on the variation of natural frequency of structure, and it is well known that the frequency variation of structural vibration is not sensitive to the change of stiffness which could be caused by structural damage. Furthermore, this FEA numerical model will be employed to simulate the numerical damages. Thus, the more accurate the FEA model is, the closer it would be between the numerical results and experimental results. Also, the conditional probability matrix evaluated through numerical simulation will be closer to that through experiments. Therefore, model updating techniques should be utilized to achieve a relative accurate FEA model. Due to implementing blind damage detection in Step 2, the model updating is merely applied to undamaged structure, that is, taken to obtain a numerical undamaged FEA model.

The model updating technique used herein is a kind of parametric method, which updates the model by minimizing the objective function that includes system parameters. Whereby, we construct an objective function $J(\mathbf{v})$ as

$$
J(\mathbf{v})=\sum_{i=1}^{N} \beta_{i}\left[f_{i}^{T}-f_{i}^{C}(\mathbf{v})\right]^{2}
$$

in which $f_{i}^{T}$ and $f_{i}^{C}$ represent the natural frequencies obtained by vibration test and FEA respectively, $N$ is the number of frequencies and is assigned a value of five, $\beta_{i}$ is the weight factor for $i$ th natural frequency, and $\mathbf{v}$ is a vector consisting of elastic module, dimensions of cantilever, and mass of accelerometers.

To minimize the objective function, stochastic research method was implemented to solve this optimization problem. Whereby, we achieved an updated model, and the 


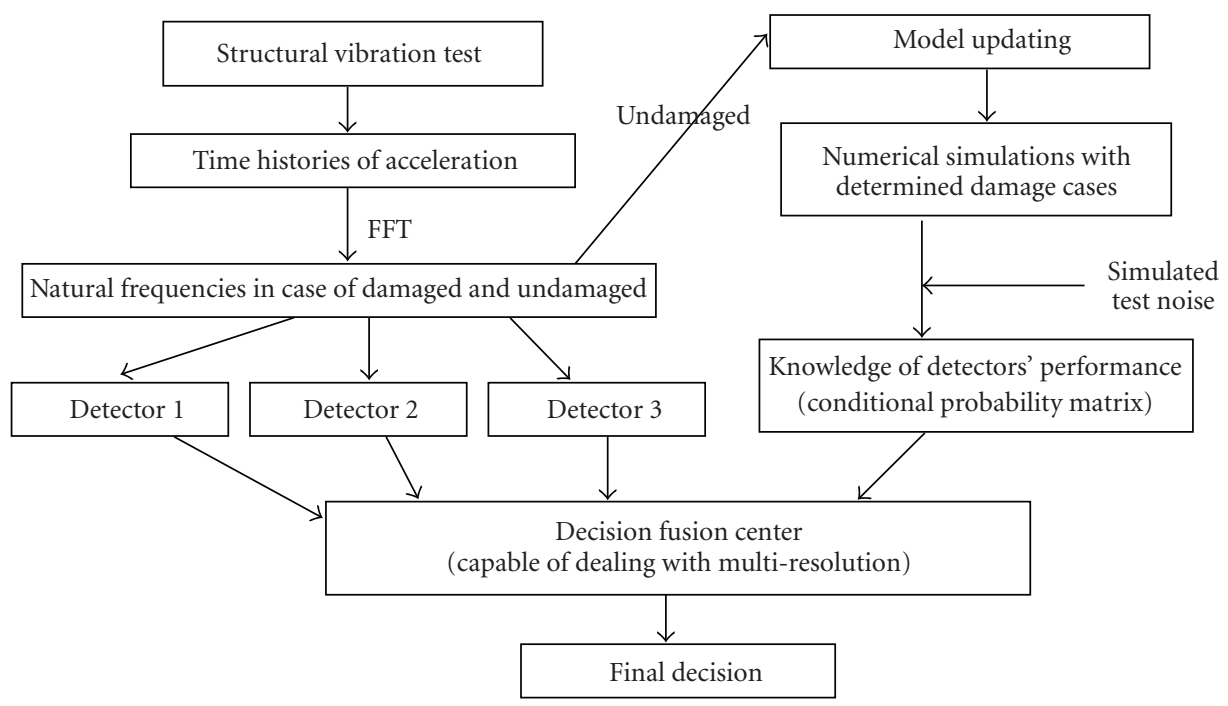

Figure 7: Decision fusion-based damage detection.
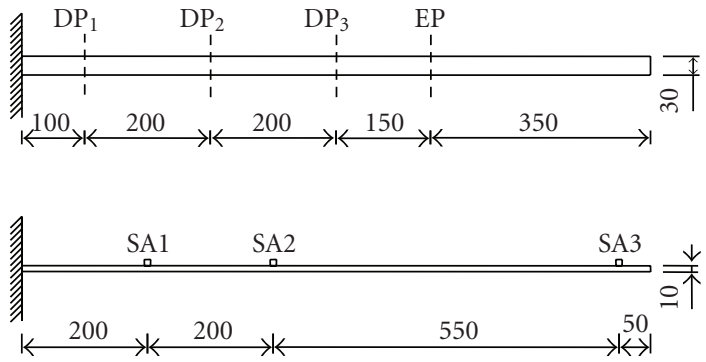

FIGURE 8: Dimensions of cantilever beam.

natural frequencies obtained through FEA are very close to those through vibration tests, as shown in Table 1.

5.2. Detection Methods. A total of three damage detection methods are employed to identify the damage in the structure. These vibration-based methods employ a linear motion system governed by

$$
\mathbf{M x}^{\prime \prime}+\mathbf{C x}^{\prime}+\mathbf{K x}=\mathbf{f}(t),
$$

where $\mathbf{M}, \mathbf{C}$ and $\mathbf{K}$ are mass, damp and stiffness matrix, respectively, and $\mathbf{x}$ is displacement vector, and $\mathbf{f}(t)$ is external excitation.

5.2.1. Damage Detection Method 1. Damage will cause the changes of both mass and stiffness matrices, and lead to a change of natural frequency $\omega$ and mode $\varphi$. According to the work by Hearn and Testa [2], if mass change and second order items can be ignored, we have the square of the $i$ th natural frequency increment, $\Delta \omega_{i}^{2}$, formed as

$$
\Delta \omega_{i}^{2}=\frac{\varphi_{i}^{T} \Delta \mathbf{K} \varphi_{i}}{\varphi_{i}^{T} \mathbf{M} \varphi_{i}} .
$$

It is assumed that the change of stiffness matrix caused by damage can be represented by the original stiffness matrix multiplied by a scalar $\alpha_{l}$. Thus, we divide the structure into $n$ elements and consider the $l$ th element, and (36) can be

$$
\Delta \omega_{i}^{2}=\frac{\alpha_{l} \varepsilon_{l}^{T}\left(\varphi_{i}\right) \mathbf{k}_{l} \varepsilon_{l}\left(\varphi_{i}\right)}{\varphi_{i}^{T} \mathbf{M} \varphi_{i}},
$$

where $\varepsilon_{l}(\varphi)$ is the element's deformation that can be calculated by changed mode due to damage. Thus

$$
\frac{\Delta \omega_{i}^{2}}{\Delta \omega_{j}^{2}}=\frac{\varepsilon_{l}^{T}\left(\varphi_{i}\right) \mathbf{k}_{l} \varepsilon_{l}\left(\varphi_{i}\right)}{\varepsilon_{l}^{T}\left(\varphi_{j}\right) \mathbf{k}_{l} \varepsilon_{l}\left(\varphi_{j}\right)} \cdot \frac{\varphi_{j}^{T} \mathbf{M} \varphi_{j}}{\varphi_{i}^{T} \mathbf{M} \varphi_{i}} .
$$

Equation (38) indicates that the ratio of any two $\Delta \omega^{2}$ would be a function of damage location, but independent of damage quantity. Hence, this method is on the second resolution level, and the detection result $u_{1}=h_{j}$ obtained is merely capable of localizing the damage or indicating no damage.

5.2.2. Damage Detection Method 2. According to Stubbs and Osegueda's work [1], for the $i$ th natural frequency, we use a variation rate $b_{i}$ to describe the ratio of $\Delta \omega_{i}^{2}$ to $\omega_{i}^{2}$ for the $i$ th natural frequency, that is,

$$
b_{i}=\frac{\Delta \omega_{i}^{2}}{\omega_{i}^{2}}=\frac{\alpha_{l} \varepsilon_{l}^{T}\left(\varphi_{i}\right) \mathbf{k}_{l} \varepsilon_{l}\left(\varphi_{i}\right)}{\varphi_{i}^{T} \mathbf{K} \varphi_{i}}
$$

It was considered that $n$ damaged elements were involved and $p$ modes were measured, therefore (39) can be rewritten as

$$
\mathbf{A}_{p \times n} \mathbf{X}_{n \times 1}=\mathbf{B}_{p \times 1},
$$

where vector $\mathbf{B}=\left[b_{1}, b_{2}, \ldots, b_{p}\right]^{T}$ consists of $p$ variation rates, vector $\mathbf{X}=\left[\alpha_{1}, \alpha_{2}, \ldots, \alpha_{n}\right]^{T}$ is damage vector whose each element is the same as $\alpha_{l}$ in (37), and matrix $\mathbf{A}$ represents the relationship matrix of damage and changing 
rate. According to (39), the elements in A can be computed by

$$
\begin{aligned}
A_{i j} & =\frac{\mathcal{\varepsilon}_{j}^{T}\left(\varphi_{i}\right) \mathbf{k}_{j} \varepsilon_{j}\left(\varphi_{i}\right)}{\varphi_{i}^{T} \mathbf{K} \varphi_{i}} \\
& =\frac{b_{i}}{a_{j}} \quad(i=1,2, \ldots, p, j=1,2, \ldots, n)
\end{aligned}
$$

The matrix A should be obtained in advance before starting damage detection, thus, numerical damage simulation is conventionally employed to obtain it. Firstly, we attain the natural frequencies of the numerical model with a damage of $\alpha_{l}$ by FEA. Then, the variation rate vector $\mathbf{B}$ can be achieved by (39). Finally, the elements of A can be computed by (41).

With knowledge of that $n<p$ may occur frequently during actual damage detection, thus (40) would lead to an undetermined system. The countermeasure suggested herein to solve (40) is to minimize an objective function $J_{s}$ as

$$
J_{s}=\frac{1}{2}\|\mathbf{A X}-\mathbf{B}\|_{2}^{2}-1 \leq \alpha_{j} \leq 0, \quad j=1, \ldots, n .
$$

Obviously, the second method is on the highest resolution level, which means that it can both localize and quantify the damage which occurred in the structure.

5.2.3. Damage Detection Method 3. The third selected method is a kind of ANN method, which utilizes neural network to identify damage. According to the achievements of Ko et al. [4], we used a three-layer BP neural network, with seven outputs representing seven damage cases, three inputs representing the first three natural frequency variation rates, and a hidden layer of eight nodes. After simulating the numerical damage, corresponding variation rates and damage cases were put into the neural network for training. Then, the trained neural network is ready for damage detection.

5.3. Damage Cases and Tests. Three damage locations are considered as shown in Figure 8, and they are named as $\mathrm{DP}_{1}$, $\mathrm{DP}_{2}$ and $\mathrm{DP}_{3}$. The damage cases for each location would be no damage, damaged with a gap in depth $\leq 3 \mathrm{~mm}$, and damaged with a gap in depth $>3 \mathrm{~mm}$. Then we achieved a total of seven damage cases or a seven hypothesis problem, as shown in Table 2.

In dynamic test, we used three test specimens to physically simulate the damages of three locations. Table 1 just lists the results of one of the specimens. For each specimen, we measured the vibrations of the undamaged beam, that is, whose gap is zero deep, and the damaged beam. The damage beam is a beam that has a gap, and the gap is sequentially cut in depth of $1,2,3,4,5$, and $6 \mathrm{~mm}$ on the side at the same location. Five repeated vibration tests were conducted for the gap in each depth, whereby, we conducted a total of 35 tests for one specimen and 105 tests for total three specimens. Then, these tests were classified according to Table 2. Whereby, 105 samples are prepared for damage identification, and the corresponding results would include the manufacturing errors and measuring errors.

\subsection{Final Decision}

5.4.1. Priori Perception. The priori perception for damage detection is the information that should be achieved before starting damage detection. As described in Step 1, the priori perception herein includes the priori probabilities of hypotheses and the conditional probability matrixes of all detectors.

For this experimental study, it is assumed that the hypotheses are equally probable, that is, the damage case obeys discrete uniform distribution. Thus, all of the priori probabilities of hypotheses are equal and each one is equal to $1 / 7$.

The conditional probability matrixes of three detectors were numerically studied. By using the updated FEA model, we also constructed the FEA models for the damage beams. The damage kind of gap is simulated through eliminating the finite elements where damage occurs. A total of 35 damage cases are simulated, and the corresponding natural frequencies are obtained through FEA. Thus by adding the pseudo experimental errors, which were numerical simulated according to the statistics of dynamic test results, to those natural frequencies, we generated lots of numerical samples. With these, three detectors were then examined, and the subdecisions obtained were counted to approximately estimate the conditional probability matrices. These matrixes are listed as

$$
\begin{aligned}
\mathbf{h}^{1} & =\left[\begin{array}{ccccccc}
84.1 & 40.2 & 0.8 & 50.3 & 0.2 & 51.6 & 0.1 \\
3.8 & 36.6 & 78.8 & 10.3 & 5.2 & 5.3 & 0 \\
5.2 & 16.2 & 18.9 & 38.5 & 94.5 & 4.5 & 0.1 \\
6.9 & 7.0 & 1.5 & 0.9 & 0.1 & 38.6 & 99.8
\end{array}\right] \%, \\
\mathbf{h}^{2} & =\left[\begin{array}{ccccccc}
34.5 & 7.6 & 0 & 3.2 & 0 & 7.5 & 0 \\
8.4 & 78.3 & 0.6 & 4.3 & 0 & 4.5 & 0 \\
0 & 2 & 99.4 & 0 & 0 & 0 & 0 \\
22.7 & 0.1 & 0 & 83.2 & 0.8 & 26.7 & 0 \\
0.1 & 0 & 0 & 3 & 99.2 & 0 & 0 \\
34.2 & 12 & 0 & 6.3 & 0 & 61.3 & 1.8 \\
0.1 & 0 & 0 & 0 & 0 & 0 & 98.2
\end{array}\right] \%, \\
\mathbf{h}^{3} & =\left[\begin{array}{ccccccc}
56 & 20 & 0 & 6.9 & 0 & 10.5 & 0 \\
3.1 & 73.3 & 13.3 & 2.3 & 0 & 18.2 & 0 \\
0.2 & 0 & 86.7 & 0 & 0 & 0 & 0 \\
25.2 & 0 & 0 & 81.3 & 4.9 & 28.7 & 0 \\
0.3 & 0 & 0 & 0.3 & 95.1 & 0 & 0 \\
15.1 & 6.7 & 0 & 9.2 & 0 & 42.6 & 2.8 \\
0.1 & 0 & 0 & 0 & 0 & 0 & 97.2
\end{array}\right] \% .
\end{aligned}
$$

However, by taking advantage of actual test results, the statistics of the sub-decisions by the detectors also provides the conditional probability matrixes of the detectors. We use an overbar to distinguish these experimental results from 
the numerical results given in (43), and these experimentally obtained matrixes are

$$
\begin{aligned}
\overline{\mathbf{h}}^{1} & =\left[\begin{array}{ccccccc}
86.7 & 33.3 & 0 & 53.3 & 0 & 66.7 & 0 \\
0 & 40 & 80 & 6.7 & 6.7 & 0 & 0 \\
6.7 & 20 & 20 & 40 & 93.3 & 0 & 0 \\
6.7 & 6.7 & 0 & 0 & 0 & 33.3 & 100
\end{array}\right] \%, \\
\overline{\mathbf{h}}^{2} & =\left[\begin{array}{ccccccc}
33.3 & 6.7 & 0 & 6.7 & 0 & 0 & 0 \\
6.7 & 80.0 & 0 & 6.7 & 0 & 0 & 0 \\
0 & 0 & 100 & 0 & 0 & 0 & 0 \\
26.7 & 0 & 0 & 80.0 & 0 & 26.7 & 0 \\
0 & 0 & 0 & 0 & 100 & 0 & 0 \\
33.3 & 13.3 & 0 & 6.7 & 0 & 73.3 & 0 \\
0 & 0 & 0 & 0 & 0 & 0 & 100
\end{array}\right] \%, \\
\overline{\mathbf{h}}^{3} & =\left[\begin{array}{ccccccc}
60 & 20 & 0 & 6.7 & 0 & 6.7 & 0 \\
0 & 73.3 & 13.3 & 0 & 0 & 20 & 0 \\
0 & 0 & 86.7 & 0 & 0 & 0 & 0 \\
26.7 & 0 & 0 & 86.7 & 0 & 26.7 & 0 \\
0 & 0 & 0 & 0 & 100 & 0 & 0 \\
13.3 & 6.7 & 0 & 6.7 & 0 & 46.7 & 0 \\
0 & 0 & 0 & 0 & 0 & 0 & 100
\end{array}\right] \% .
\end{aligned}
$$

Comparing the conditional probability matrixes from numerical simulations with those from tests, only a small difference could be observed. Accordingly, it is reasonable to use the conditional probability matrices from the numerical analysis results. Usually, the performance of the detector is not available, experience from applications is absent, and dynamic tests for damaged structure could not be realized, in civil engineering. Therefore, this numerical simulationbased method is useful to achieve those conditional probability matrixes.

5.4.2. Damage Detections by Detectors and Fusion. With the priori probability of hypotheses as introduced in the Section 5.4.1, and the detectors' performance matrix as listed in (43), the priori perception for this experimental study of decision fusion are prepared now. Whereby, (7) is capable of achieving a final decision while the sub-decisions are made. It is noted that the sub-decisions made by the detectors for a damage case should be based on the actual test results, because we are dealing with blind damage detection.

Firstly, for each one of 105 tests in Section 5.3, all of three damage detection methods should make a decision to determine the damage case, according to the experimental results from that test. Then, by the use of priori perception and (7), the sub-decisions were fused to achieve a final decision about damage case. It is noted, because the detectors employed herein are in different resolutions, the fusion strategy for multiresolution should be implemented.

The Selected 6 samples with corresponding three damage detection results $u_{i}(i=1,2,3)$ and final decision $u$ are listed in Table 3, in which the value is the damage case number. The actual damage cases represented by $H$ are also presented in Table 3, for comparison.

It is shown that a decision fusion system can make a more reliable decision, compared with using single damage detection method. It often happens that the final decision conforms well to the actual damage case but conflicts occur
TABLE 2: Damage cases denoted according to damage location and degree.

\begin{tabular}{lccccccc}
\hline $\begin{array}{l}\text { Damage } \\
\text { case no. }\end{array}$ & $H_{0}$ & $H_{1}$ & $H_{2}$ & $H_{3}$ & $H_{4}$ & $H_{5}$ & $H_{6}$ \\
\hline $\begin{array}{l}\text { Damage } \\
\text { location }\end{array}$ & None & DP1 & DP1 & DP2 & DP2 & DP3 & DP3 \\
$\begin{array}{l}\text { Damage } \\
\text { degree }\end{array}$ & $\leq 3 \mathrm{~mm}>3 \mathrm{~mm}$ & $\leq 3 \mathrm{~mm}$ & $>3 \mathrm{~mm}$ & $\leq 3 \mathrm{~mm}$ & $>3 \mathrm{~mm}$ \\
\hline
\end{tabular}

TABLE 3: Selected samples and fusion result.

\begin{tabular}{lcccccc}
\hline Samples no. & $H$ & $u_{1}$ & $u_{2}$ & $u_{3}$ & $u$ & Correct or Wrong \\
\hline 1 & 0 & 0 & 0 & 0 & 0 & $\sqrt{ }$ \\
2 & 0 & 0 & 3 & 0 & 0 & $\sqrt{ }$ \\
3 & 0 & 2 & 0 & 5 & 0 & $\sqrt{ }$ \\
4 & 1 & 0 & 1 & 0 & 1 & $\sqrt{ }$ \\
5 & 1 & 3 & 5 & 4 & 0 & $\times$ \\
6 & 1 & 2 & 1 & 1 & 1 & $\sqrt{ }$ \\
\hline
\end{tabular}

among three damage detectors. After applying the decision fusion to the results of 105 vibration tests, we also achieved a conditional probability matrix, as shown in (45), which shows superiority to that of any one of the detectors.

$$
\overline{\mathbf{h}}^{u}=\left[\begin{array}{ccccccc}
80 & 13.3 & 0 & 20 & 0 & 26.7 & 0 \\
6.7 & 66.7 & 0 & 0 & 0 & 0 & 0 \\
0 & 20 & 100 & 0 & 0 & 0 & 0 \\
0 & 0 & 0 & 80 & 0 & 0 & 0 \\
0 & 0 & 0 & 0 & 100 & 0 & 0 \\
13.3 & 0 & 0 & 0 & 0 & 73.3 & 0 \\
0 & 0 & 0 & 0 & 0 & 0 & 100
\end{array}\right] \%
$$

\section{Conclusion}

Decision fusion method can integrate individual damage detection measures to obtain a single measure, and achieve a higher probability of correct selection than any individual one. This paper introduced the implementation of decision fusion in structural damage detection. The MJP decision fusion rule was employed to deal with multiple hypotheses detection, fusing sub-decisions in multiple resolutions, and blind adaptive detection. An implementation using experimental structure was conducted. The whole detection and fusion process were illustrated.

MJP method relies on the priori perception of system, including priori probability of damage cases and conditional probability matrixes of detectors. Therefore, we introduced a method to obtain the conditional probability matrix of detectors by FEA method. This method was approved by comparing the matrixes from numerical simulation with those from test results. A cantilever beam structure was set up for experimental study, and 105 vibration test samples were obtained for damage detection. Then, three vibrationbased detectors were employed to make sub-decisions based on test results. In terms of priori perceptions and these subdecisions, a final decision can be obtained by using the MJP decision fusion rule. Thus, 105 final decisions were achieved, 
and it was found that the final decisions were superior to those from a single damage detection method.

\section{References}

[1] N. Stubbs and R. Osegueda, "Global non-destructive damage evaluation in solids-experimental verification," The International Journal of Analytical and Experimental Modal Analysis, vol. 5, pp. 81-97, 1990.

[2] G. Hearn and R. B. Testa, "Modal analysis for damage detection in structures," Journal of Structural Engineering, vol. 117, pp. 3042-3063, 1991.

[3] Y. Zou, L. Tong, and G. P. Steven, "Vibration-based modeldependent damage (delamination) identification and health monitoring for composite structures-a review," Journal of Sound and Vibration, vol. 230, no. 2, pp. 357-378, 2000.

[4] J. M. Ko, Y. Q. Ni, X. T. Zhou, and J. Y. Wang, "Structural damage alarming in Ting Kau Bridge using auto-associative neural networks," in Advances in Structural Dynamics, J. M. Ko and Y. L. Xu, Eds., vol. 2, pp. 1021-1028, Elsevier Science, Oxford, UK, 2000.

[5] Z. Sun and C. C. Chang, "Structural damage assessment based on wavelet packet transform," Journal of Structural Engineering, vol. 128, no. 10, pp. 1354-1361, 2002.

[6] A. Sinha, H. Chen, D. G. Danu, T. Kirubarajan, and M. Farooq, "Estimation and decision fusion: a survey," Neurocomputing, vol. 71, no. 13-15, pp. 2650-2656, 2008.

[7] R. R. Tenney and N. R. Sandell Jr., "Detection with distributed sensors," IEEE Transactions on Aerospace and Electronic Systems, vol. 17, no. 4, pp. 501-510, 1981.

[8] Z. Chair and P. K. Varshney, "Optimum data fusion in multiple sensor detection systems," IEEE Transactions on Aerospace and Electronic Systems, vol. 22, no. 1, pp. 98-101, 1986.

[9] K. Demirbas, "Maximum a Posteriori approach to object recognition with distributed sensors," IEEE Transactions on Aerospace and Electronic Systems, vol. 24, no. 3, pp. 309-313, 1988.

[10] W. Baek and S. Bommareddy, "Optimal m-ary data fusion with distributed sensors," IEEE Transactions on Aerospace and Electronic Systems, vol. 31, no. 3, pp. 1150-1152, 1995.

[11] N. Ansari, J.-G. Chen, and Y.-Z. Zhang, "Adaptive decision fusion for unequiprobable sources," IEE Proceedings on Radar, Sonar and Navigation, vol. 144, no. 3, pp. 105-110, 1997.

[12] N. Mansouri and M. Fathi, "Simple counting rule for optimal data fusion," in Proceedings of the IEEE Conference on Control Applications, vol. 2, pp. 1186-1191, 2003.

[13] B. Liu, A. Jeremic, and K. M. Wong, "Blind adaptive algorithm for M-ary distributed detection," in Proceedings of the IEEE International Conference on Acoustics, Speech and Signal Processing (ICASSP '07), vol. 2, pp. 1025-1028, 2007.

[14] M. N. Liu and X. Yang, "Image quality assessment by decision fusion," IEICE Electronics Express, vol. 5, no. 15, pp. 537-542, 2008.

[15] H. Seraji and N. Serrano, "A multisensor decision fusion system for terrain safety assessment," IEEE Transactions on Robotics, vol. 25, no. 1, pp. 99-108, 2009.

[16] A. Kyriazis and K. Mathioudakis, "Gas turbine fault diagnosis using fuzzy-based decision fusion," Journal of Propulsion and Power, vol. 25, no. 2, pp. 335-343, 2009.

[17] H. Guo and L. Zhang, "A weighted balance evidence theory for structural multiple damage localization," Computer Methods in Applied Mechanics and Engineering, vol. 195, no. 44-47, pp. 6225-6238, 2006.
[18] C. Zhou, Y. Chen, and B. Sun, "Result-merging algorithm of structure damage identifications with multi-resolution based on D-S evidential theory," Journal of Vibration and Shock, vol. 25, no. 6, pp. 5-8, 2006 (Chinese).

[19] H. Li, Y. Bao, and J. Ou, "Structural damage identification based on integration of information fusion and shannon entropy," Mechanical Systems and Signal Processing, vol. 22, no. 6, pp. 1427-1440, 2008.

[20] C. Tshöpe and M. Wolff, "Statistical classifiers for structural health monitoring," IEEE Sensors Journal, vol. 9, no. 11, pp. 1567-1576, 2009.

[21] S.-Y. Tian, Y. Chen, and B.-N. Sun, "Maximum joint probability fusion rule for multi-resolution decision of structure damage detection," Journal of Zhejiang University (Engineering Science), vol. 41, no. 1, pp. 126-133, 2007 (Chinese).

[22] X. Wang, G. Foliente, Z. Su, and L. Ye, "Multilevel decision fusion in a distributed active sensor network for structural damage detection," Structural Health Monitoring, vol. 5, no. 1, pp. 45-58, 2006.

[23] J. K. Ghosh, M. Delampady, and T. Samanta, An Introduction to Bayesian Analysis: Theory and Methods, Springer, New York, NY, USA, 2006. 

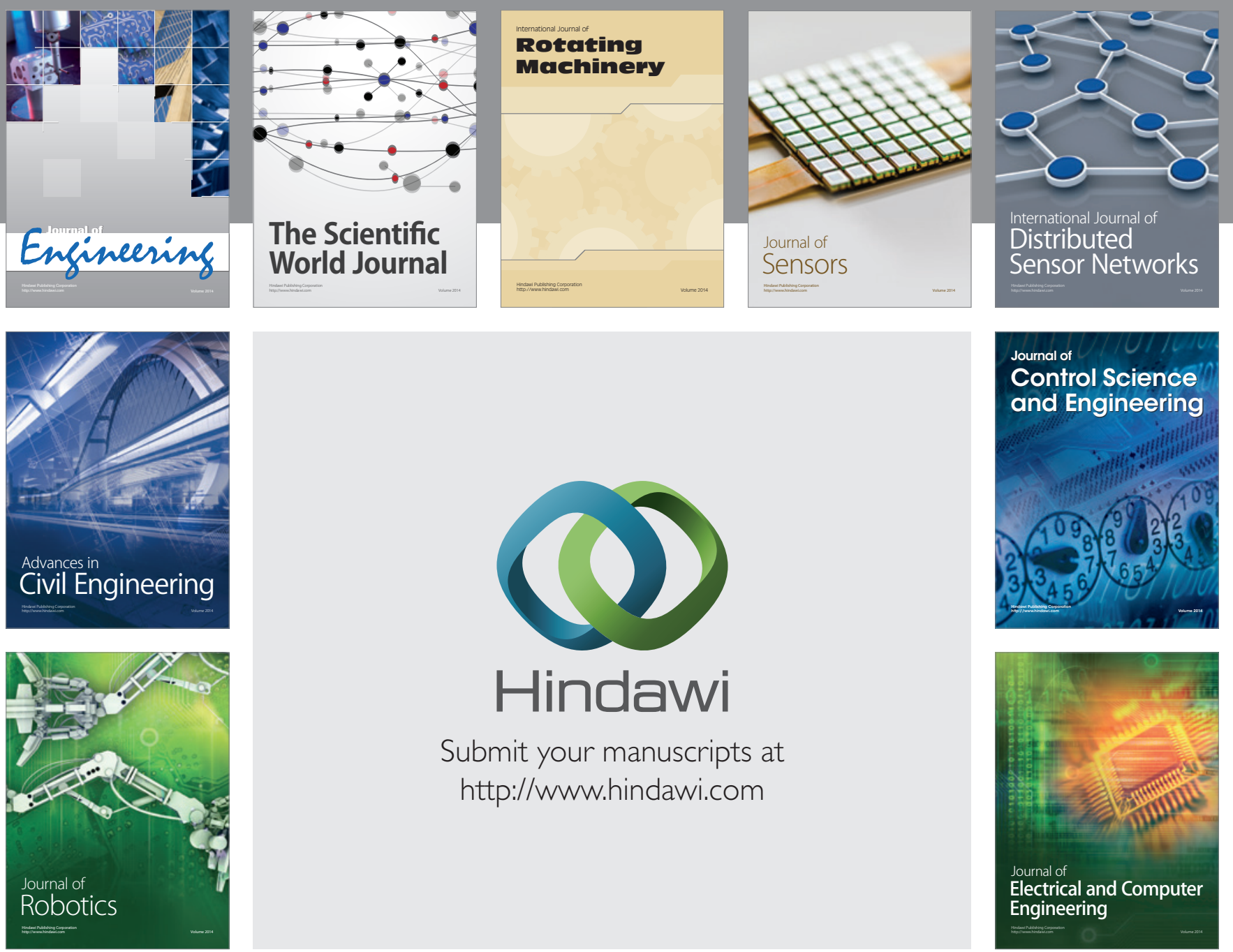

Submit your manuscripts at

http://www.hindawi.com
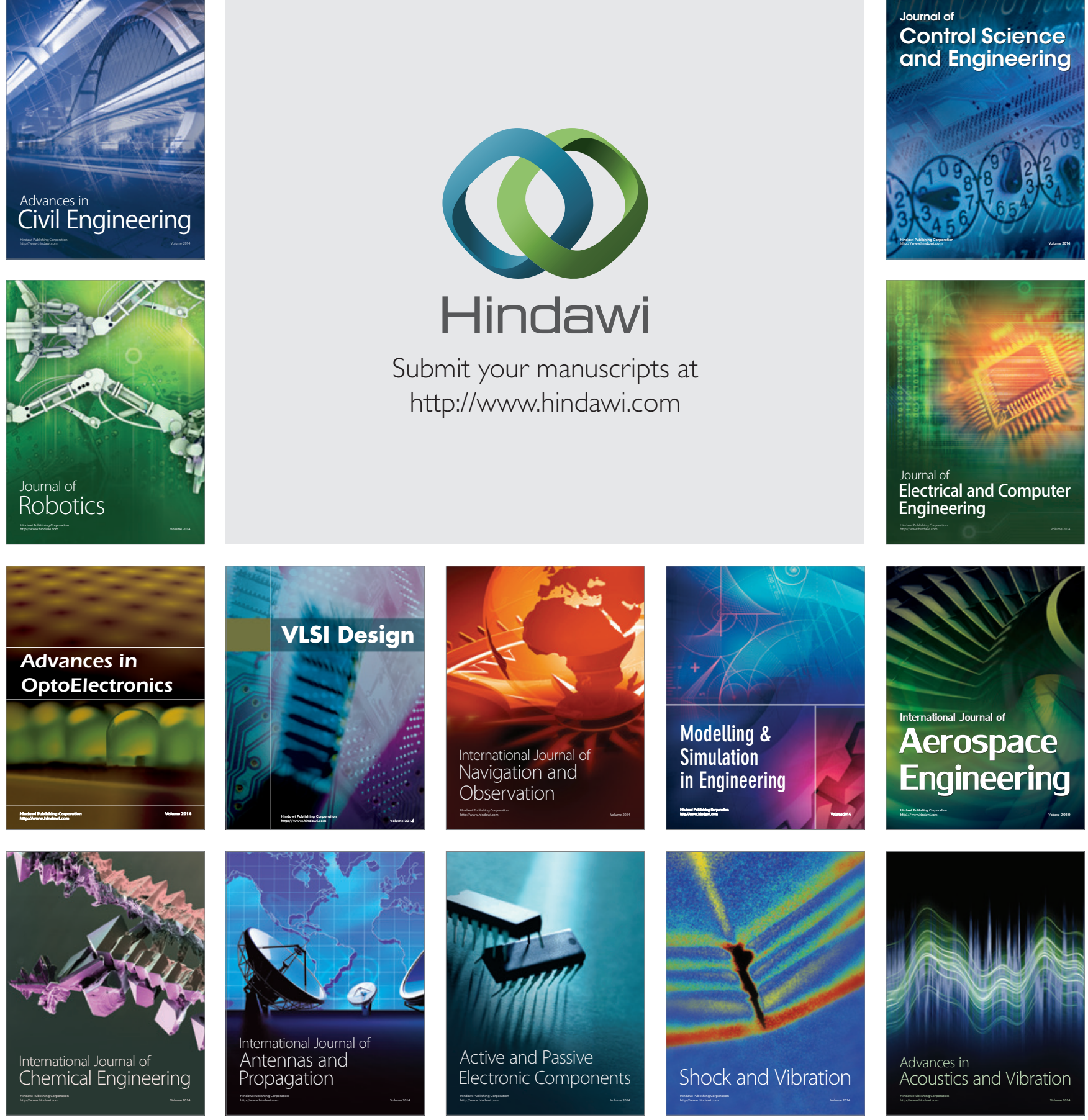